\title{
Comparación entre las Técnicas de Ricketts y McNamara para la Determinación de la Posición del Maxilar y la Mandíbula en Jóvenes del Ecuador
}

\author{
Comparison between Ricketts and McNamara Techniques for the Determination \\ of the Maxilla and Mandible Position in Ecuadorian Youths
}

\author{
Marco Guerrero $^{1,2}$; Juan Ocampo ${ }^{1,3}$ \& Sergio Olate Su,5 $^{1,45}$
}

GUERRERO, M.; OCAMPO, J. \& OLATE, S. Comparación entre las técnicas de Ricketts y McNamara para la determinación de la posición del maxilar y la mandíbula en jóvenes del Ecuador. Int. J. Morphol., 36(1):169-174, 2018.

RESUMEN: Los análisis cefalométricos, a partir de una radiografía lateral de cráneo, son amplios y variados considerando tanto los patrones funcionales como los de estética facial. Entre las técnicas de medición en cefalometría, Ricketts y de McNamara son ampliamente utilizados; ambas le entregan importancia a determinar la posición del maxilar y de la mandíbula con diferentes relaciones esqueletales. El objetivo de esta investigación es comparar ambas técnicas en una población joven de Ecuador; se realizó un análisis de concordancia utilizando el índice kappa de Cohen entre los resultados de las mediciones correspondientes a 44 radiografías cefálicas laterales de sujetos jóvenes entre 18 y 27 años de edad, de ambos sexos. En relación a la posición maxilar, el 56,82 \% (IC 95 \%: 42,18 \% - 71,45 \%) coinciden en el diagnóstico de protrusión maxilar; el índice de concordancia kappa de Cohen fue de 0,599 (IC 95\%: 0,354 - 0,854) con un valor p<0,05. En relación a la posición mandibular, el 50,0 \% (IC $95 \%: 35,23 \%$ - 64,77 \%) coinciden en el diagnóstico de retrusión mandibular; el índice kappa de Cohen fue de 0,460 (IC $95 \%$ 0,229 - 0,691) con un valor p<0,05. Se concluye que, a pesar de manejar puntos de referencia cefalométricos diferentes, las dos técnicas de medición cráneo-facial son útiles para el estudio de posición de maxila y mandíbula. Existen diferencias entre algunos analisis de ambos estudios que pueden incidir en la selección terapéutica de potenciales pacientes.

PALABRAS CLAVE: Morfología facial; Cefalometría; Deformidad Facial.

\section{INTRODUCCIÓN}

Los estudios cefalométricos para el estudio de la forma y caracteristicas del componente cráneo-maxilomandibular, son muy utilizados en el anáisis de sujetos con deformidades faciales y en la correlación con algunas patologías, como la apnea del sueño (Borges et al., 2015) y la calcificaciones de tejidos blandos (Garay \& Olate, 2013), entre otras. Sujetos con respiración bucal por obstrucción de vía aérea superior muestran cambios en los análisis, por ejemplo, en el ángulo maxilomandibular (Pulido Valladares et al., 2012). Igualmente, estos estudios son empleados con frecuencia en la planificación y seguimiento de rehabilitación oral, demostrando su versatilidad (Carrera Vidal et al., 2010; Castro Arenas et al., 2013).

En el estudio de las maloclusiones, la aplicación de la cefalometría es muy importante (Martínez et al., 2008;
Gómez Gómez et al., 2011; Martínez-Arriagada et al., 2105). Se considera que el análisis cefalométrico es de gran utilidad para el diagnóstico de deformidades faciales, deformidades craneo maxilo mandibulares y deformidades dentales, en las cuales el posicionamiento maxilar y mandibular presentan una especial importancia (Nunes et al., 2015).

En términos de la morfología facial, determinada por este tipo de estudios, se han encontrado diferencias sustanciales entre los parámetros estandarizados para caucásicos y para mestizos de la zona peruana (Menéndez Méndez, 2009). En este sentido, se han realizado diversos perfiles raciales cefalométricos, incluso estéticos, que fueron inicialmente de origen anglosajón, pero que en la actualidad provienen de otros distintos lugares, como Japón, China, India, algunos países de África y, en América Latina, en Chile,

\footnotetext{
${ }^{1}$ Programa de Doctorado en Ciencias Morfológicas, Universidad de La Frontera, Temuco, Chile.

${ }^{2}$ Cátedra de Anatomía Normal. Facultad de Ciencias Médicas. Universidad Central del Ecuador, Quito, Ecuador.

${ }^{3}$ Cátedra de Histología Normal. Facultad de Ciencias Médicas. Universidad Central del Ecuador, Quito, Ecuador.

${ }^{4}$ División de Cirugía Oral, Facial y Maxilofacial, Universidad de La Frontera, Temuco, Chile.

${ }^{5}$ Centro de Excelencia en Estudios Morfológicos y Quirurgicos, Universidad de La Frontera, Chile.
} 
Cuba, Brasil, Colombia, México y Bolivia, entre otros (Conde Suárez et al., 2013; De la Rosa et al., 2013; Bronfman et al., 2015; Cuenca Tigreros et al., 2015; Montt et al., 2015).

Según Vargas Flores \& Vargas Flores (2012), los resultados cefalométricos están asociados con la edad, sexo, raza-etnia y otras características, fisiológicas o no, del sujeto de estudio. Las técnicas de medición cefalométrica son variadas, siendo algunas de ellas las de Steiner, Ricketts y McNamara, los que han sido empleados durante los ultimos 40 años (Carrera Vidal et al.; Cuenca Tigreros et al.).

El análisis de Steiner es uno de los primeros utilizados en la cefalometría moderna y algunos de sus elementos siguen vigentes. El análisis de Ricketts es más amplio, permite la informatización de los datos, maneja varias incidencias, decenas de puntos y diez factores descriptivos; le otorga gran relevancia a la relación maxilar-mandibular, mediante las mediciones de N-Pog y su relación con el punto A y Xi-Pog (Carrera Vidal et al.). El análisis de McNamara se realiza a partir de la medición de otras estructuras y constituye una buena referencia para evaluar el maxilar y la mandíbula en relación a la base de cráneo. Su propósito principal es obtener diferencias entre los componentes esqueletales y dento-alveolares utilizando el triángulo denominado con el mismo nombre (Co-A, Co-Gn, ENA-Me) (Vargas Flores $\&$ Vargas Flores).

En la era de los estudios 3D, el uso de una imagen 2D (telerradiografía) en estos estudios implica algunas ventajas como la de presentar menos costos y menos exposición a la radiación (da Silva \& Sant'Anna et al., 2013). Estudios, como el de Esteva Segura et al. (2014), demuestran que la correcta aplicación de las mediciones mediante trazos manuales en los trabajos cefalométricos, son igualmente precisos que la utilización de un programa computacional junto a radiografías digitales.

El objetivo de esta investigación es establecer el nivel de concordancia entre dos técnicas de medición cefalométrica (de Ricketts y de McNamara) para la determinación de la posición del maxilar y de la mandíbula en jóvenes estudiantes universitarios del Ecuador.

\section{MATERIAL Y MÉTODO}

En el presente estudio se utilizaron 44 teleradiografías laterales. Se incluyeron sujetos de ambos sexos y de entre 18 y 27 años de edad, estudiantes universitarios seleccionados por conveniencia (Carrera de Medicina de la Universidad Central del Ecuador, en Quito). Las teleradiografías fueron tomadas en un solo centro radiológico especializado, con el equipo radiográfico marca SODEREX, modelo Cranex (Finlandia, 2012); para la obtención de la imagen, el sujeto fue posicionado de acuerdo a protocolos previamente establecidos, manteniendo estandarización en los procesos; se excluyeron sujetos que presentaran malformaciones faciales, experiencia de trauma maxilofacial previo y sujetos con deformidades faciales asimétricas.

En las imágenes se realizaron los trazados correspondientes para determinar la posición del maxilar y la mandíbula según la técnica de Ricketts, considerando la línea N-Pog-punto A para determinar posición maxilar (parámetro
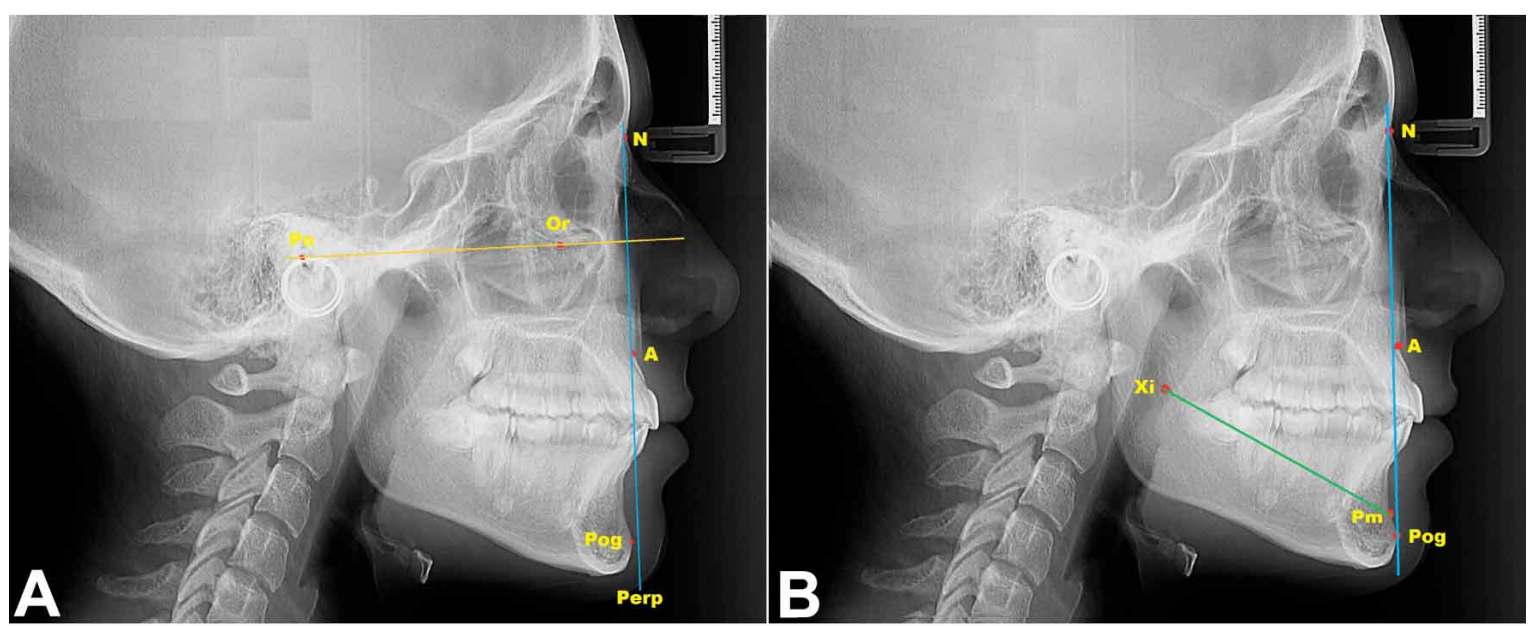

Fig. 1. Radiografías cefálicas laterales para cefalometría de McNamara y Ricketts en la evaluación de la posición anteroposterior maxilar y mandibula. A: Análisis de McNamara que utiliza el plano de Frankfurt (línea Po-Or) para el trazo de la línea N-perpendicular-punto A para evaluación de la posición anteroposterior del maxilar, N-perpendicular-Pog para evaluación de la posición anteroposterior de la mandíbula. B: Análisis de Ricketts que utiliza la línea N-Pog para evaluación de la posición anteroposterior del maxilar, y el trazo Xi-Pm para evaluar la longitud de la mandíbula. 
de normalidad: $0 \mathrm{~mm} \pm 2 \mathrm{~mm}$ ) y la línea Xi-Pm para determinar longitud mandibular (parámetro de normalidad: 81 $\mathrm{mm} \pm 3 \mathrm{~mm}$ ); de la técnica de McNamara utilizamos la línea N-perpendicular-punto A para posición maxilar (parámetro de normalidad: $1 \mathrm{~mm}$ ) y la línea N-perpendicular-Pog para la posición cefalométrica de la mandíbula (parámetro de normalidad: $2 \mathrm{~mm}$ a $4 \mathrm{~mm}$ ). Se dibujó sobre papel vegetal el perfil blando y óseo con énfasis en los puntos cefalométricos específicos de acuerdo a cada técnica (Fig. 1).

Posteriormente, se utilizó el programa estadístico SPSS 24® para el procesamiento de los datos, obteniendo frecuencias absolutas y relativas de los diagnósticos y utilizando tablas de contingencia para comparar los mismos según las mediciones de acuerdo a los parámetros establecidos para las técnicas en estudio. Se obtuvo el índice kappa de Cohen con su respectivo intervalo de confianza al $95 \%$, para medir la concordancia de la posición maxilar y mandibular entre las técnicas. Para la prueba de hipótesis trabajamos con un error alfa de menos de 0,05.

\section{RESULTADOS}

La distribución de frecuencias de la posición maxilar y mandibular según las técnicas de Ricketts y McNamara muestra un mayor porcentaje de maxilares protruidos, considerando en los estudios de Ricketts un 61,4\% (IC $95 \%$ : $42,45 \%$ - 80,27 \%) y en los de McNamara un 70,5\% (IC 95 $\%: 52,74 \%$ - 88,17\%); en cambio para la posición mandibular la técnica de Ricketts muestra una mayor frecuencia para mandíbula retruida con un 59,1\% (IC $95 \%$ : $40,0 \%-78,18 \%$ ) y la técnica de McNamara muestra una mayor frecuencia de mandíbulas de posición normal con un 61,4\% (IC $95 \%$ : 42,45 \% - 80,27\%) (Tabla I).

Al comparar las técnicas de Ricketts y McNamara para evaluar la posición maxilar se observó que en el diagnóstico de protrusión maxilar las dos técnicas fueron útiles en la identificación, considerando un 56,82\% de los sujetos (IC $95 \%$ : 42,18\% - 71,45\%) (Tabla II).

Tabla I. Distribución de frecuencias de la evaluación de la posición maxilar y mandibular entre las técnicas de Ricketts y McNamara, en jóvenes estudiantes de 18 a 27 años de edad.

\begin{tabular}{lcccccc}
\hline & \multicolumn{3}{c}{ Técnica de Ricketts } & \multicolumn{2}{c}{ Técnica de McNamara } \\
\hline Posición & $\mathrm{n}$ & $\%$ & IC 95\% & $\mathrm{n}$ & $\%$ & IC 95\% \\
Maxilar protruido & 27 & 61,4 & $42,45-80,27$ & 31 & 70,5 & $52,74-88,17$ \\
Maxilar retruido & 0 & 0,0 & - & 0 & 0,0 & - \\
Maxilar normal & 17 & 38,6 & $19,73-57,54$ & 13 & 29,5 & $11,83-47,26$ \\
Mandíbula protruida & 0 & 0,0 & - & 7 & 15,9 & $1,71-30,11$ \\
Mandíbula retruida & 26 & 59,1 & $40,0-78,18$ & 10 & 22,7 & $6,45-39,0$ \\
Mandíbula normal & 18 & 40,9 & $21,82-60,0$ & 27 & 61,4 & $42,45-80,27$ \\
\hline
\end{tabular}

Tabla II. Tabla de contingencia para análisis de concordancia entre las técnicas de Ricketts y McNamara en la evaluación de la posición maxilar en jóvenes estudiantes de 18 a 27 años de edad.

\begin{tabular}{|c|c|c|c|c|c|c|}
\hline \multirow{3}{*}{$\begin{array}{l}\text { Posición del maxilar según } \\
\text { Técnica de McNamara }\end{array}$} & \multicolumn{6}{|c|}{ Posición maxilar según técnica de Ricketts } \\
\hline & \multicolumn{2}{|c|}{ Normal } & \multicolumn{2}{|c|}{ Protrusión maxilar } & \multicolumn{2}{|c|}{ Total } \\
\hline & $\mathrm{n}$ & $\%$ & $\mathrm{n}$ & $\%$ & $\mathrm{n}$ & $\%$ \\
\hline Normal & 11 & 25,00 & 6 & 13,64 & 13 & 29,55 \\
\hline Protrusión maxilar & 2 & 4,55 & 25 & 56,82 & 31 & 70,45 \\
\hline Total & 11 & 25,00 & 33 & 75,00 & 44 & 100,00 \\
\hline
\end{tabular}


Para estas medidas el nivel de concordancia es mediano de acuerdo con el valor del índice kappa de Cohen ponderado obtenido de 0,599 (IC $95 \% 0,354$ a 0,844), sin embargo este valor es significativamente distinto que el esperado por el azar $(\mathrm{p}<0,05)$.

Respecto de la posición mandibular observamos que las dos técnicas han logrado clasificar con el diagnóstico de retrusión mandibular al 50,0 \% (IC 95 \%: 35,23\% - 64,77 $\%)$ de la población estudiada. (Tabla III). El nivel de concordancia también es mediano de acuerdo con el valor del índice kappa de Cohen ponderado obtenido de 0,450 (IC 95 $\% 0,229$ a 0,691 ); este valor es significativamente distinto al que se podría esperar simplemente por el azar $(\mathrm{p}<0,05)$.

Tabla III. Tabla de contingencia para análisis de concordancia entre las técnicas de Ricketts y McNamara en la evaluación de la posición mandibular en jóvenes estudiantes de 18 a 27 años de edad.

\begin{tabular}{lcccrcr}
\hline & \multicolumn{2}{c}{ Posición de la man díbula según técnica de Ricketts } \\
Posición de la mandíbula & \multicolumn{2}{c}{ Normal } & \multicolumn{2}{c}{$\begin{array}{c}\text { Retrusión } \\
\text { mandibular }\end{array}$} & \multicolumn{2}{c}{ Total } \\
\cline { 2 - 7 } según téc nica de McNamara & $\mathrm{n}$ & $\%$ & $\mathrm{n}$ & $\%$ & $\mathrm{n}$ & $\%$ \\
\hline Normal & 10 & 22,73 & 1 & 2,27 & 12 & 27,27 \\
Retrusión mandibular & 8 & 18,18 & 22 & 50,00 & 2 & 4,55 \\
Protrusión mandibular & 1 & 2,27 & 2 & 4,55 & 30 & 68,18 \\
Total & 21 & 47,73 & 23 & 52,27 & 44 & 100,00 \\
\hline
\end{tabular}

\section{DISCUSIÓN}

El diagnóstico de sujetos con patología creaneofacial tradicionalmente se ha realizado con tecnología 2D utilizando cefalogramas bidimensionales (2D); bajo esa metodología se han desarrollado la mayoría de las investigaciones (San Miguel de Moragas et al., 2014; San Miguel de Moragas et al., 2015; Olate et al., 2016); sin embargo, las mismas investigaciones han demostrado variabilidad en resultados toda vez que la condición tridimensional (3D) del rostro limitan el consenso y precisión en los análisis 2D.

Swennen et al. (2006) indicaron que los estudios 2D son factibles de presentar sesgo en términos de relación entre tejidos duros y blandos; sin embargo, los estudios 3D no han mostrado claridad en los resultados presentados y se han observado múltiples sesgos en las investigaciones publicadas que aumentan las limitaciones observadas en los estudios (Olate et al., 2017).

La cefalometría de McNamara tiene como objetivo principal realizar un analisis y finalmente un diagnóstico de la morfología y posición de diferentes estructuras del esqueleto facial, estableciendo una relación entre el componente maxilar, el componente mandibular y la base del cráneo; en los estudios de Ricketts, por otra parte, se valora la posición de estos mismos huesos, independientemente de la base del cráneo, fundamentándose en la estética y armonía ósea facial (De la Rosa et al.; Cárdenas et al., 2016).
Considerando que las dos técnicas tienen parámetros de normalidad basados en individuos caucásicos, en la evaluación obtenida en esta investigación, existe una mayor frecuencia de protrusión maxilar y retrusión mandibular, según los análisis. Algunos autores definen diferencias de los patrones de normalidad observados en los valores numéricos que presentan sujetos caucásicos y los obtenidos de otras razas-etnias, como la mestiza (Menéndez Méndez; Cuenca Tigreros et al.; De la Rosa et al.); en un estudio realizado en una población autóctona del estado de México se examinaron 100 radiografías de niños de ambos sexos de 6 a 13 años observando un $64 \%$ de retrusión mandibular, bajo parametros comparativos de normalidad de tipo anglosajón (Álvarez et al, 2006), de forma que cabe la interrogante de cual es la real normalidad de posición del esqueleto facial en diferentes etnias. En nuestro estudio, por ejemplo, solo con la técnica de Ricketts es posible observar una mayor frecuencia de retrusión mandibular.

Comparando ambas técnicas fue posible observar diferencias porcentuales entre los diagnósticos. Por ejemplo, la mandibula retrudida fue observada con mayor frecuencia en los análisis de Ricketts (casi un $40 \%$ mas que en los resultados presentados por McNamara) y la maxila protruida, por el contrario, fue mas frecuente en los estudios de McNamara (casi $10 \%$ mayor a los datos de 
Rocketts); estas diferencias pueden ser explicadas por los puntos basales de los análisis, donde McNamara utiliza un plano Po-Or para establecer una línea estable desde la que se permita analizar posiciones en base a la relación craneal, lo que no es observado en los estudios de Ricketts.

Otra posible explicación es que algunos puntos necesarios para el análisis son de difícil ubicación y registro, tales como el porion anatómico; además, la distancia entre N-per y el punto A, en los casos de una excesiva inclinación anterior de las raíces de los incisivos superiores podría limitar la exactitud de las mediciones (Fernández \& Da Silva, 2009).

A pesar de las diferencias, el análisis estadístico realizado mediante el índice kappa de Cohen permitió establecer la concordancia entre las dos técnicas para evaluar la posición maxilar y mandibular (Carrasco \& Jover, 2004). En ambos casos hubo una mediana concordancia, estadísticamente significativa, determinando algunos hallazgos no normales según los valores de referencia de las técnicas, tanto en la posición de maxila como en mandíbula.

GUERRERO, M.; OCAMPO, J. \& OLATE, S. Comparison between Ricketts and McNamara techniques for the determination of the maxilla and mandible position in Ecuadorian youths. Int. J. Morphol., 36(1):169-174, 2018.

SUMMARY: The cephalometric analyses from a lateral cranial radiograph, are wide and varied considering both functional and facial aesthetic patterns. Among the measurement techniques in cephalometry, Ricketts and McNamara are widely used; they both give importance to determine the position of the maxilla and mandible with different skeletal relationships. The objective of this research is to compare both techniques in a young population of Ecuador. A concordance analysis was performed using Cohen's kappa index between the results of the measurements corresponding to 44 lateral cephalic radiographs of young subjects between 18 and 27 years of age, of both sexes. In relation to the maxillary position, $56.82 \%$ (95\% CI: $42.18 \%-71.45 \%$ ) coincide in the diagnosis of maxillary protrusion. Cohen's kappa concordance index was 0.599 (95\% CI: 0.354-0.854) with a p-value < 0.05 . In relation to the mandibular position, $50.0 \%$ (95\% CI: $35.23 \%$ $64.77 \%$ ) coincide in the diagnosis of mandibular retrusion. Cohen's kappa index was 0.460 (95\% CI 0.229 - 0.691) with a p-value < 0.05 . It is concluded that, despite handling different cephalometric reference points, the two craniofacial measurement techniques are useful for the study of maxilla and mandible position. There are differences between some analyses of both studies that may affect the therapeutic selection of potential patients.

KEY WORDS: Facial morphology; Cephalometry; Facial deformity.

\section{REFERENCIAS BILIOGRÁFICAS}

Álvarez, N. C. A.; Servín, H. S. V. \& Parés, V. F. Frecuencia de los componentes de la maloclusión clase II esquelética en dentición mixta. Rev. Asoc. Dent. Mex., 63(6):210-4, 2006.

Borges, P. de T.; Silva, B. B.; Moita Neto, J. M.; Borges, N. E. \& Li, L. M. Cephalometric and anthropometric data of obstructive apnea in different age groups. Braz. J. Otorhinolaryngol., 81(1):79-84, 2015.

Bronfman, C. N.; Janson, G.; Pinzan, A. \& Rocha, T. L. Cephalometric norms and esthetic profile preference for the Japanese: a systematic review. Dental Press J. Orthod., 20(6):43-51, 2015.

Cárdenas, J. M.; Bañuelos, J. M. G.; Meraz, W. S.; Murga, H. M.: Cárdenas, G. M.; Navarro Rincón-Gallardo, M. E. \& Cantú, F. J. G. Transversal comparative study of mcnamara maxillofacial mandibular ratio applied in Mexican subjects. Int. J. Morphol., 34(2):454-9, 2016.

Carrasco, J. L. \& Jover, L. Métodos estadísticos para evaluar la concordancia. Med. Clin., 122 Supl. 1:28-34, 2004.

Carrera Vidal, C.; Larrucea Verdugo, C. \& Galaz Valdés, C. Detección de incrementos de dimensión vertical oclusal mediante análisis cefalométrico de Ricketts. Rev. Clin. Periodoncia Implantol. Rehabil. Oral, 3(2):79-85, 2010.

Castro Arenas, M. V.; Hurtado Peralta, M. \& Oyonarte Weldt, R. Rendimiento de la evaluación cefalométrica para el diagnóstico sagital intermaxilar. Revisión narrativa. Rev. Clin. Periodoncia Implantol. Rehabil. Oral, 6(2):99-104, 2013.

Conde Suárez, H. F.; González, F. V. \& Sánchez Gay, J. M. Normas del cefalograma de Ricketts en niños de Matanzas. Rev. Cuba. Estomatol., 50(4):388-96, 2013.

Cuenca Tigreros, D.; Caicedo Cruz, M.; Bedoya Rodriguez, A.; Osorio, J. C. \& Martínez Cajas, C. Medidas cefalométricas aplicadas en estudios para caracterización de poblaciones determinadas: Revisión sistemática. Rev. Colomb. Investig. Odontol., 6(16):16-40, 2015.

da Silva, M. B. \& Sant'Anna, E. F. The evolution of cephalometric diagnosis in orthodontics. Dental Press J. Orthod., 18(3):63-71, 2013.

De la Rosa, C. A. V.; Montiel, B. N. M.; Kubodera, I. T. \& Jasso, R. I. Elaboración de un estándar cefalométrico para la población del centro de la República Mexicana, mayor de 15 años de edad, basado en el análisis craneofacial de Ricketts. Rev. Asoc. Dent. Mex., 70(5):251-7, 2013.

Esteva Segura, F. J.; Sánchez Valverde, A.; Meléndez Ocampo, A. \& Cedillo Angelares, P. R. Estudio comparativo entre la cefalometría digital y manual con radiografías digitales. Rev. Mex. Ortodon., 2(2):95-8, 2014.

Fernández, J. \& Da Silva, O. Atlas Cefalometría y Análisis Facial. Madrid, Editorial Ripano, 2009.

Garay, I. \& Olate, S. Currrent considerations in the study of image of soft tissue calcification in mandibular angle area. Int. J. Odontostomat., 7(3):455-64, 2013.

Gómez Gómez, V.; Fernández López, A. \& Pérez Tejada, H. E. Características cefalométricas presentes en la maloclusión clase I en el departamento de Ortodoncia de la DEPeI. Rev. Odontol. Mex., 15(1):14-20, 2011.

Martínez-Arriagada, V.; Schulz-Rosales, R.; Cerda-Peralta, B.; RiveraRothgaenger, M.; López-Garrido, J.; Mora-Figueroa, N. \& RomoOrmazábal, F. Paralelismo entre el plano oclusal y 3 planos cefalométricos. Rev. Clin. Periodoncia Implantol. Rehabil. Oral, 8(3):234-8, 2015.

Martínez, G. R.; Mendoza, O. L.; Fernández, L. A. \& Pérez, T. H. E. Características cefalométricas en la maloclusión clase II. Rev. Odontol. Mex., 12(1):7-12, 2008.

Menéndez Méndez, L. Estudio comparativo entre mestizas y caucásicos mediante el análisis cefalométrico de Ricketts. Odontol. Sanmarquina, 12(2):66-9, 2009.

Montt, R. J.; Miquel, V. M. P. \& Oyonarte, W. R. Cephalometric characteristics in young individuals with normal occlusion and harmonic 
GUERRERO, M.; OCAMPO, J. \& OLATE, S. Comparación entre las técnicas de Ricketts y McNamara para la determinación de la posición del maxilar y la mandíbula en jóvenes del Ecuador. Int. J. Morphol., 36(1):169-174, 2018.

profiles in chilean population. Int. J. Morphol., 33(1):237-44, 2015.

Nunes, H. J.; Jardim, E. C. G.; Oliveira, M. M.; Masocatto, D. C.; dos Santos, C. M.; Quadros, D. C.; Hassumi, J. S. \& de Mendonça, J. C. G. Análise cefalométrica: uma nova proposta para diagnóstico de deformidades dento-esqueletais. Braz. J. Surg. Clin. Res., 12(1):27-37, 2015.

Olate, S.; Zaror, C. \& Mommaerts, M. Y. A systematic review of soft-tohard tissue ratios in orthognathic surgery. Part IV: 3D analysis - Is there evidence? J. Craniomaxillofac. Surg., 45(8):1278-86, 2017.

Olate, S.; Zaror, C.; Blythe, J. N. \& Mommaerts, M. Y. A systematic review of soft-to-hard tissue ratios in orthognathic surgery. Part III: Double jaw surgery procedures. J. Craniomaxillofac. Surg., 44(10):1599-606, 2016.

Pulido Valladares, Y.; Piloto Morejón, M.; Gounelas Amat, S.; Rezk Díaz, A. \& Duque Alberro, Y. Cambios cefalométricos en pacientes respiradores bucales con obstrucción de vías aéreas superiores entre 812 años. Rev. Cienc. Med., 16(5):90-103, 2012.

San Miguel de Moragas, J.; Oth, O.; Büttner, M. \& Mommaerts, M. Y. A systematic review on soft-to-hard tissue ratios in orthognathic surgery part II: Chin procedures. J. Craniomaxillofac. Surg., 43(8):1530-40, 2015.

San Miguel de Moragas, J.; Van Cauteren, W. \& Mommaerts, M. Y. A systematic review on soft-to-hard tissue ratios in orthognathic surgery part I: maxillary repositioning osteotomy. J. Craniomaxillofac. Surg., 42(7):1341-51, 2014.

Swennen, G. R.; Schutyser, F.; Barth, E. L.; De Groeve, P. \& De Mey, A. A new method of 3-D cephalometry Part I: the anatomic Cartesian 3-D reference system. J. Craniofac. Surg., 17(2):314-25, 2006.

Vargas Flores, T. I. \& Vargas Flores, T. C. Parámetros cefalométricos en adultos de oclusión normal. Rev. Actual. Clin. Med., 20(2):997-1002, 2012.

\section{Dirección para correspondencia \\ Prof. Dr. Sergio Olate \\ Facultad de Odontología \\ Universidad de La Frontera \\ Claro Solar 115, oficina 414-A \\ CHILE}

E-mail: sergio.olate@ufrontera.cl

Recibido : 27-10-2017

Aceptado: 27-11-2017 